\title{
Around the mandible
}

\section{Hee-Jin Kim • Jean-Michel Prades • Bruno Grignon - Fabrice Duparc}

Published online: 9 October 2014

(C) Springer-Verlag France 2014

After the 2013 special issue devoted to the cranial nerves and the face, numerous studies about the lower part of the face were submitted, and we proposed to continue in this field of clinical anatomy, and to propose this 2014 topic "Around the mandible".

Through this title, we aimed to consider the bone itself, the very particular course of nerves within intra-osseous canals, the foramina entering and exiting of the nerves, the imaging for depicting these structures, and some variations. Moreover, the clinical expression of the pathologies of the mandible or of the temporomandibular joint justified to include studies about this joint. To support the difficult diagnosis of pain in this area, a regional and rare case of variation is exposed.

At last, specific anatomical conditions as edentulate patients may lead to remodeling the mandible and change the anatomical features.

We hope you will enjoy this short travel around the mandible.

\section{H.-J. Kim}

Division in Anatomy and Developmental Biology, Department of Oral Biology, Yonsei University College of Dentistry, 250

Seongsanno, Seodaemoon-gu, Seoul 120-752, Korea

\section{J.-M. Prades}

Laboratory of Anatomy, Faculty of Medicine, Saint Etienne University, 15 Rue Ambroise Paré, 42023 Saint Etienne Cedex, France

\section{B. Grignon}

Département d'Anatomie, Faculté de Médecine de Nancy,

Université de Lorraine, Nancy Cedex, France

\section{B. Grignon}

Service d'Imagerie Guilloz, Hôpital Central, CHU Nancy, 29,

Avenue du Maréchal de Lattre de Tassigny CO 34,

54035 Nancy, France

F. Duparc $(\bowtie)$

Laboratory of Anatomy, Faculty of Medicine, Rouen University,

22 Bld Gambetta, 76183-1 Rouen, France

e-mail: fabrice.duparc@univ-rouen.fr 